\title{
Extreme events induced by spatiotemporal chaos in experimental optical patterns
}

\author{
Marcel G. Clere, ${ }^{1}$ Gregorio González-Cortés, ${ }^{1}$ and Mario Wilson ${ }^{1,2, *}$ \\ ${ }^{1}$ Departamento de Física, Facultad de Ciencias Físicas y Matemáticas, Universidad de Chile, Blanco Encalada 2008, Santiago, Chile \\ ${ }^{2}$ Current address CONACYT-CICESE, Carretera Ensenada-Tijuana 3918, Zona Playitas, C.P. 22860, Ensenada, Mexico \\ *Corresponding author: mwilson@cicese.mx
}

Received 16 March 2016; revised 16 May 2016; accepted 16 May 2016; posted 18 May 2016 (Doc. ID 261286); published 6 June 2016

Extreme events such as rogue waves are often associated with the merging of coherent structures. We report on experimental results in the physics of extreme events emerging in a liquid-crystal light valve subjected to optical feedback, and we establish the relation of this phenomenon with the appearance of spatiotemporal chaos. This system, under particular conditions, exhibits stationary roll patterns that can be destabilized into quasi-periodic and chaotic textures when control parameters are properly modified. We have identified the parameter regions where extreme fluctuations of the amplitude can emerge and established their origin through its direct relation with the experimental largest Lyapunov exponents, the proportion of extreme events, and the normed kurtosis. (c) 2016 Optical Society of America

OCIS codes: (190.0190) Nonlinear optics; (190.3100) Instabilities and chaos; (030.6600) Statistical optics; (230.3720) Liquid-crystal devices.

http://dx.doi.org/10.1364/OL.41.002711

A time record generated by a natural system may consist of well-defined periods, where a relevant variable undergoes small variations around a well-defined level provided by its long-time average, with the occasional appearance of abrupt excursions to higher values that differ significantly from the average level; these excursions are usually called extreme events [1]. Extreme and rare events are ubiquitous in nature; these kinds of phenomena can appear in a wide variety of contexts, including hydrodynamics [2], plasma physics [3], optics [4,5], earthquakes [6], and weather [7]. The study of extreme and rogue events has been motivated in analogy to rogue waves in hydrodynamics [8]; these rogue waves are giant waves that have been observed in the ocean and whose mechanisms are not yet fully established. One well-established approach states that the creation of these extreme events is caused by the collision of coherent structures, mainly modeled by the nonlinear Schrödinger equation [4]. Recently, most of the studies in this way have been performed in optical fibers, where the interplay between nonlinearity, noise, and dispersion generates extreme pulses, reinforcing that the creation mechanism is based on the merging dynamics of coherent structures [9-12]. More recently, extreme events have been reported in nonlinear optical cavities [13,14], solid-state lasers [15], photorefractive crystals [16], laser diodes with phase-conjugation [17], and extended microcavity lasers [18]. Experimental observations of extreme events in optics are mainly concentrated in the statistical characterization of this phenomenon. Few studies are concentrated in the determination of the undergoing mechanisms of creation that cause these events. One different approach states that extreme events are created by the interplay of stochastic processes in systems presenting multistability; pointing the noise creates intermittence, noise-induced transition, and acts as a switch between coexisting non-chaotic states [19]. A deterministic mechanism in non-extended systems has been established based on the emergence of chaos through a crisis [20]. For extended systems, it has recently been established numerically that the emergence of spatiotemporal chaos expedites the appearance of extreme events [18]. More precisely, the statistical characterization is based on experimental observation. Nevertheless, the spatiotemporal chaos is established by its Lyapunov spectrum which is obtained through numerical simulations in a generic model.

In this Letter, we experimentally show that the emergence of extreme events in the amplitude fluctuations is directly related to the emergence of spatiotemporal intermittency of stripe patterns. Based on a liquid crystal light valve subjected to optical feedback, which exhibits a transition from stationary roll patterns to spatiotemporal chaotic textures, we study the dynamics of the amplitude fluctuations. The presence of intermittency between different dynamical regimes, i.e., spatiotemporal chaos and quasi-periodicity, allows a huge energy exchange, giving rise to extreme and giant events.

A flexible experimental setup that exhibits pattern formation in nonlinear optics is the liquid-crystal light valve (LCLV) with optical feedback [21]. Particularly, in this setup, regular patterns such as hexagonal, stripe and zig-zag structures, super lattices, localized structures, dislocations, disclinations, spiral states, domain walls between patterns, front propagation, and quasi-crystals, have been reported (see [21] and references therein). This setup contains a LCLV inserted in an optical feedback system (see Fig. 1). The LCLV is composed of a nematic liquid-crystal film sandwiched between a glass and a photoconductive plate over which a dielectric mirror is 


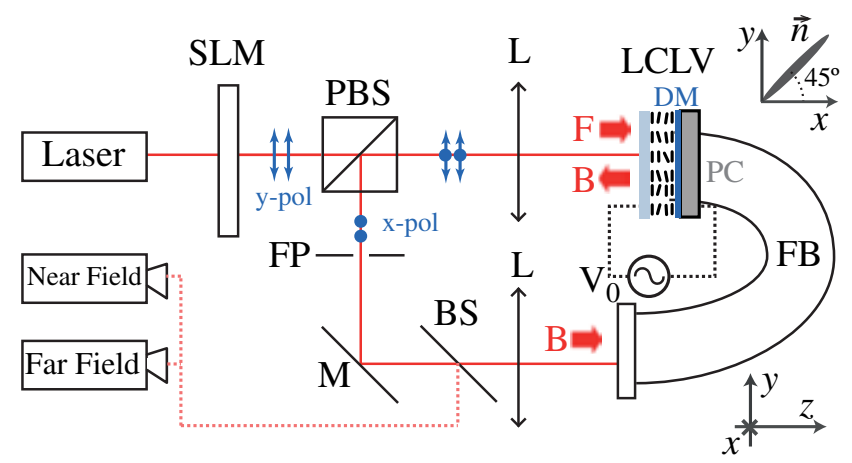

Fig. 1. Schematic representation of the experimental setup. LCLV, $\mathrm{L}$, two lenses with a focal distance $f=25 \mathrm{~cm} ; \mathrm{M}$, mirror; $\mathrm{FB}$, optical fiber bundle; PBS, polarizing beam splitter cube; BS, beam splitter; FP, Fourier plane; PC, photoconductor layer; DM, dielectric mirror; SLM, spatial light modulator driven by a computer. $V_{0}$ external voltage applied across the LCLV. F and B represent the forward and the backward beams, respectively.

deposed. The liquid-crystal film is planar aligned (nematic director $\vec{n}$ parallel to the walls), with a thickness $d=15 \mu \mathrm{m}$. The liquid-crystal (LC) filling our LCLV is a nematic LC-654, produced by NIOPIK (Moscow) [22]. It is a mixture of cyano-biphenyls, with a positive dielectric anisotropy $\Delta \epsilon=$ $\epsilon_{\|}-\epsilon_{\perp}=10.7$ and large optical birefringence, $\Delta n=n_{\|}-$ $n_{\perp}=0.2$, where $\epsilon_{\|}$and $\epsilon_{\perp}$ are the dielectric permittivities \| and $\perp$ to $\vec{n}$, respectively; $n_{\|}$and are $n_{\perp}$ are the extraordinary (\| to $\vec{n})$ and ordinary ( $\perp$ to $\vec{n})$ refractive index [23]. Transparent electrodes over the glass plates permit the application of an electrical voltage across the LC layer. The photoconductor behaves like a variable resistance, which decreases for increasing illumination. The feedback is obtained by sending back onto the photoconductor, through a fiber bundle (FB), the light which has passed through the liquid-crystal layer and has been reflected by the dielectric mirror. This light beam experiences a phase shift which depends on the liquid crystal orientation and, on its turn, modulates the effective voltage that locally applies to the liquid crystal sample. Transparent electrodes deposited over the cell walls allow an external voltage application $V_{0}$ across the LC layer. Over a critical voltage, molecules tend to orient along the direction of the applied electric field, which, on its turn, changes locally and dynamically by following the illumination spatial distribution present in the photoconductor wall of the cell. When LC molecules reorient, due to their birefringent nature, they induce a refractive index change. Thus, the LCLV acts as a manageable Kerr medium, causing a phase variation $\phi=2 k d \Delta n \cos ^{2} \theta$ in the reflected beam proportional to the intensity of the beam $I_{w}$ incoming on the photoconductive side, where $\theta$ is the longitudinal average of molecular reorientation. Here, $k=2 \pi / \lambda$ is the optical wave number. A schematic picture of the performed experiment is depicted in Fig. 1.

The LCLV is illuminated by an expanded He-Ne laser beam, $\lambda=633 \mathrm{~nm}$, with $1 \mathrm{~cm}$ transverse diameter and power $I_{\text {in }}=6.5 \mathrm{~mW} / \mathrm{cm}^{2}$, linearly polarized along the vertical $y$-axis. The dielectric mirror (DM)-located in the rear part of the cell-reflects the incident light in the LCLV and sends it back to the polarizing beam splitter (PBS). The PBS will send the $\mathrm{x}$-polarized light into the feedback loop. To close the feedback loop, a mirror (M) and an optical FB are used; these elements assure the light to reach the back part (photoconductor, PC) of the valve. The PBS samples and combines on the $x$-axis the ordinary and the extraordinary waves propagating in the LC layer, thus introducing interference between them. This produces a $\phi$ dependent amplitude modulation of the reflected beam which, sent on the feedback loop, ensures bistability between differently orientated states of the LC molecules. Here the optical axis of the LCLV is oriented at $45^{\circ}$ with respect to the $y$-axis $(x-y$ plane) such that the modulation depth is maximized [21]. In the feedback loop, a 4-f array is placed to obtain a self-imaging configuration and access to the Fourier plane (FP); this array is constructed with two identical lenses (L) with a focal length $f=25 \mathrm{~cm}$ placed in such a way that both sides of the LCLV are conjugated planes. Thanks to this configuration, the free propagation length in the feedback loop can be easily adjusted. For the performed experiments, a length of $d=-4 \mathrm{~cm}$ was used. A spatial light modulator (SLM) was placed in the input beam optical path with a $1: 1$ imaging between the SLM and the frontal part of the LCLV to homogenize the illuminated area and to control the boundary conditions. The SLM is a LC display with 1 inch diagonal size and $1024 \times 768$ pixel resolution; each pixel is coded in 8 bits of intensity level, and the whole system is controlled through an external computer. With the aid of specialized software, a square mask was produced in the SLM, which acts as a programmable filter able to impose arbitrary border conditions to the input beam. For a uniform mask of 160 gray value, the typical input intensity would be $I_{w}=0.83 \mathrm{~mW} / \mathrm{cm}^{2}$. To obtain the shape used in the experiments, a two-dimensional mask, $I(x, y)$, was created with an adequate shape. The system dynamics is controlled by adjusting the external voltage $V_{0}$ applied to the LCLV.

In brief, the dynamics exhibited by the LCLV with optical feedback, is that the liquid-crystal molecular orientation changes the phase of light emerging from the LCLV which, due to the optical feedback, induces a voltage that reorients the liquid-crystal molecules (see details in [21,24]). Therefore, thanks to the optical circuit, the LC molecular orientation self-induces a non-local spatiotemporal dynamics.

To explore the conditions where extreme events can occur in the proposed system, the LCLV was illuminated with the aid of an intensity mask of zero-level intensity everywhere, except for a central square part with a length $2.5 \mathrm{~mm}$. The SLM output intensity is spatially modulated as $I_{\text {in }}=I_{0}(x, y)$, where $I_{0}$ can be controlled by changing the mask values and $\{x, y\}$ are the transverse coordinates. $I_{0}$ is measured when a given gray value is imposed to the illuminated area; that is,

$$
I_{0}(x, y)=\left\{\begin{array}{ll}
I_{0} & |x| \leq a_{0}, \\
0 & \text { else. }
\end{array} \text { and } \quad|y| \leq a_{0} .\right.
$$

In the used configuration, $I_{0}=0.9 \mathrm{~mW} / \mathrm{cm}^{2}$. This average intensity was constant along all the conducted experiments. $V_{0}$ varies between 0 and $7 V_{\text {rms }}$. The critical voltage, $V_{0}=3.5 V_{\text {rms }}$, corresponds to the destabilization threshold for stationary roll patterns. In the performed experiments, 5000 images, each one of $640 * 480$ pixels weighted by 256 gray intensity levels, were recorded for each $V_{0}$ value. An adequate algorithm has been created to detect and save spatial peaks in each image. According to the literature, to consider an event as extreme, the adopted statistical criterion is through the abnormality index (AI). This criterion was defined to study 
extreme events in the ocean [2]. The AI is defined as the significant peak height corresponding to the average peak height among one-third of the highest peaks of the whole temporal series. An event is considered extreme if its amplitude is larger than twice the AI. Figure 2 presents the probability density function (PDF) as a function of the AI and snapshot for three different control parameter $V_{0}$ values. Figure 2(a) shows the PDF for the case when $V_{0}$ forces the system to enter in a regime where destructive interference starts, meaning that the maximum reached value will be small, around 50 in a 256 scale, as can be seen in Fig. 2(b). In Figs. 2(c) and 2(e), the PDF for two different $V_{0}$ values are plotted, and their respective snapshots are depicted in Figs. 2(d) and 2(f). From the insets in Figs. 2(c) and 2(e), a difference between PDF shapes can be noted; in Fig. 2(c), an L-shape curve is observed, meaning the curve presents a clear deviation from a Gaussian distribution, while in Fig. 2(e) a Gaussian decay is observed. The $L$-shape curve has been associated with the existence of rogue waves, i.e., waves that posses an extremely high amplitude respect to the average [4]. One important fact is that extreme events appear randomly across the illuminated area; Fig. 3 shows the spatial location of the highest peak for each frame, always being an extreme event. The most common AI for the highest peak of each frame is between 4.5 and 5.5. The dynamics of the system under study has been recently experimentally characterized by the authors $[25,26]$, who found that this system exhibits different dynamical regimes, including quasi-periodicity, intermittency, and spatiotemporal chaos. Using the experimental (a)

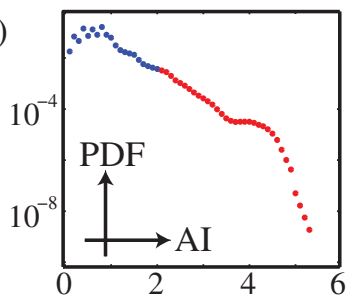

(c)

10

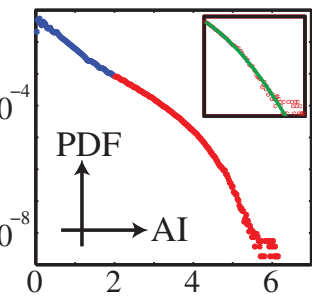

(e)

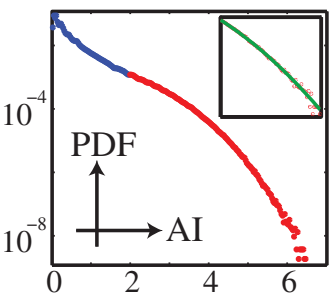

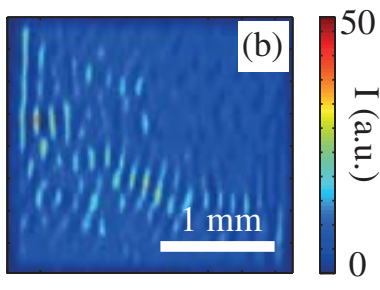
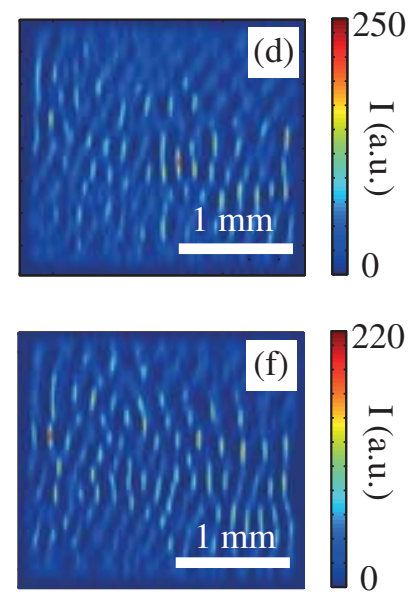

Fig. 2. Experimental extreme events. The left panel PDF as a function of the abnormality index (AI). The small insets show tails versus a Gaussian fit represented by the solid line (green). In the right panel, snapshots for three different voltages $V_{0}$ are presented. (a) and (b) correspond to $V_{0}=3.60 V_{\text {rms }}$, (c) and (d) stand for $V_{0}=4.35 V_{\text {rms }}$, and (e) and (f) stand for $V_{0}=5.90 V_{\text {rms. }}$. The gray (blue) dots stand for non-extreme events, i.e., with $A I \leq 2$; the black (red) dots show the extreme events, which $A I>2$.

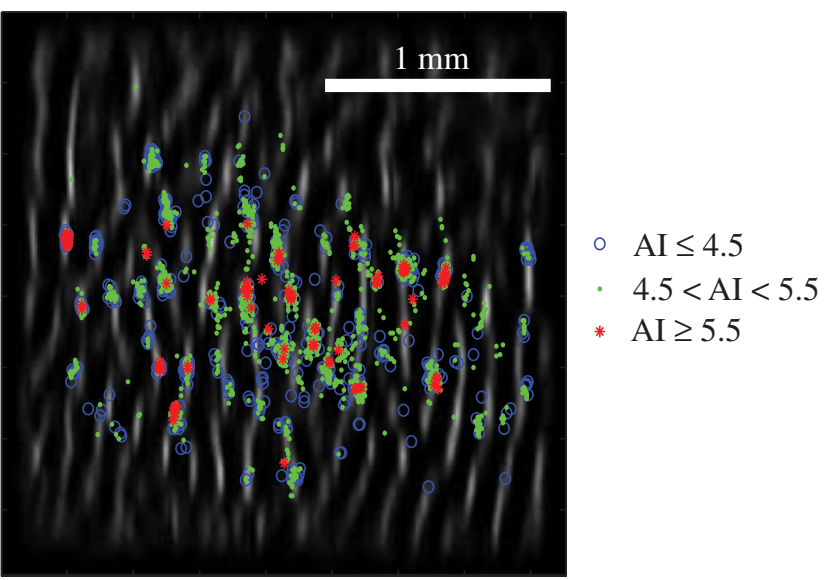

Fig. 3. Location of maxima. The position of the maximum value for each frame is spatially distributed along the texture, $V_{0}=4.7 V_{\mathrm{rms}}$. The circles correspond to $A I \leq 4.5$, the points stand for $4.5<A I<5.5$, and the stars stand for values in the range $A I \geq 5.5$. The background image is an arbitrary frame only for spatial reference.

largest Lyapunov exponents, $\lambda_{0}$, a bifurcation diagram can be constructed to show the different dynamical regimes, as can be seen in the upper panel of Fig. 4; in the lower panel, two additional measurements are plotted, the normed kurtosis, $\gamma$, and the proportion of extreme events, $P_{\mathrm{EE}}$. It can be noted that the $P_{\mathrm{EE}}$ is directly correlated with $\gamma$.

The highest kurtosis (and $P_{\mathrm{EE}}$ ) regime is located in the quasi-periodic window; this is due to the fewer quantity of modes existing in a quasi-periodic regime compared with a chaotic one, i.e., the energy is split into few possible peaks with bigger amplitudes. Nevertheless, the classical $L$-shape associated to rogue events is, in our case, produced by intermittency behaviors, as can be seen in Fig. 2(e), due to the energy exchange caused by the jumps between different dynamical regimes that the system is visiting. Note that the spatiotemporal

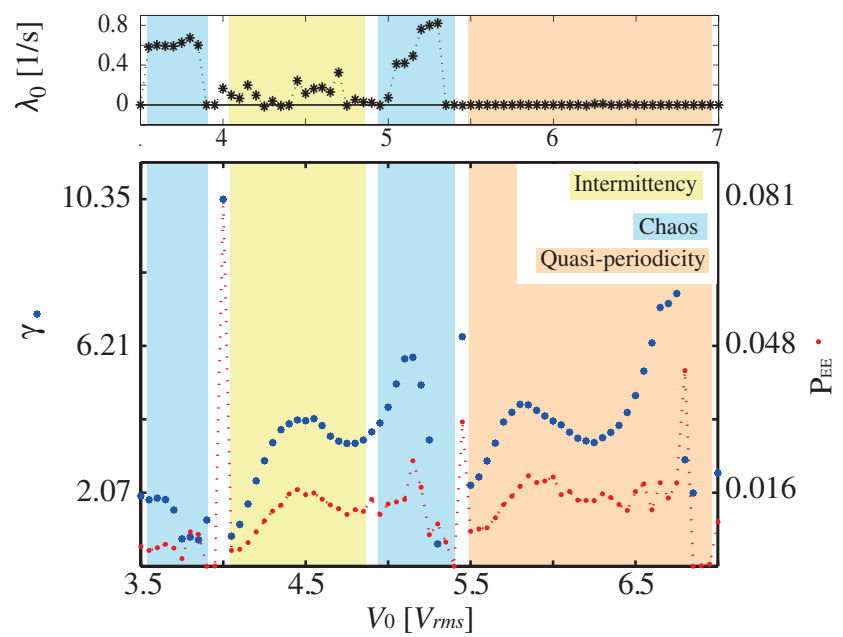

Fig. 4. Experimental largest Lyapunov exponents $\left(\lambda_{0}\right)$ plotted in the upper part. The lower part, in gray (blue), shows normed kurtosis $(\gamma)$; the black (red) portion shows the proportion of extreme events $\left(P_{\mathrm{EE}}\right)$ versus control voltage $\left(V_{0}\right)$. As the voltage $V_{0}$ is increased, the dynamical behavior of the system changes, passing through intermittency, chaos, and quasi-periodicity. 
intermittency is a consequence that a region of phase space becomes hyperbolic, allowing the system exhibits long excursions, events of large amplitude fluctuations, which return to the hyperbolic region, ghost region, staying in these regions for long periods, before making a new great excursion. Such intermittent behavior fundamentally contributes to extreme events. A mechanism that generates spatiotemporal intermittent behaviors is a crisis, that is, a stabilization of a strange object. The characteristic signature of a crisis is the appearance of the largest positive Lyapunov exponent discontinuously (see Fig. 4). This type of dynamic behavior is robust or structurally stable due to the hyperbolic nature of the strange object. Hence, one expects a large region of parameters where this phenomenon is observed.

In summary, we have reported on rare and extreme fluctuations of the amplitude in a 2-D pattern forming optical system based on a LCLV subjected to simple optical feedback. In a highly nonlinear regime, where the system exhibits intermittency and spatiotemporal chaos, this system presents high localized intensity peaks. Using well-established criteria, as normed kurtosis, proportion of extreme events, and abnormality factor, we proved that these peaks possess the signature of spatial rogue waves. Using the experimental largest Lyapunov exponents of the system, we can explain the high kurtosis windows, and we can define the role of intermittency and spatiotemporal chaos in the appearance of extreme events. Finally, relating the formation of freak waves with a well-defined dynamical regime, e.g., spatiotemporal chaos, intermittency, or quasi-periodicity, makes it easy to understand the mechanism of creation of extreme and rogue waves, suggesting that a valid way to generate these events is deterministic instead of stochastic; nevertheless, the control of these kinds of structures remains challenging.

Funding. Fondo Nacional de Desarrollo Científico y Tecnológico (FONDECYT) (1150507, 3140387).

Acknowledgment. The authors thank R. Barboza for technical support.

\section{REFERENCES AND NOTES}

1. G. Nicolis and C. Nicolis, Foundations of Complex Systems: Emergence, Information and Prediction, 2nd ed. (World Scientific, 2012).
2. C. Kharif, E. Pelinovsky, and A. Slunyaev, Rogue Waves in the Ocean (Springer, 2009).

3. H. Bailung, S. K. Sharma, and Y. Nakamura, Phys. Rev. Lett. 107, 255005 (2011).

4. D. R. Solli, C. Ropers, P. Koonath, and B. Jalali, Nature 450, 1054 (2007).

5. C. Liu, R. E. C. van der Wel, N. Rotenberg, L. Kuipers, T. F. Krauss, A. DiFalco, and A. Fratalocchi, Nat. Phys. 11, 358 (2015).

6. D. Sornette, L. Knopoff, Y. Y. Kagan, and C. Vanneste, J. Geophys. Res. Solid Earth 101, 13883 (1996).

7. K. E. Trenberth, J. T. Fasullo, and T. G. Shepherd, Nat. Clim. Change 5, 725 (2015).

8. C. Kharif and E. Pelinovsky, Eur. J. Mech. B. Fluids 22, 603 (2003).

9. J. M. Dudley, F. Dias, M. Erkintalo, and G. Genty, Nat. Photonics 8, 755 (2014).

10. A. Mussot, A. Kudlinski, M. Kolobov, E. Louvergneaux, M. Douay, and M. Taki, Opt. Express 17, 17010 (2009).

11. B. Kibler, J. Fatome, C. Finot, G. Millot, F. Dias, G. Genty, N. Akhmediev, and J. M. Dudley, Nat. Phys. 6, 790 (2010).

12. F. T. Arecchi, U. Bortolozzo, A. Montina, and S. Residori, Phys. Rev. Lett. 106, 153901 (2011).

13. A. Montina, U. Bortolozzo, S. Residori, and F. T. Arecchi, Phys. Rev. Lett. 103, 173901 (2009).

14. A. K. D. Bosco, D. Wolfersberger, and M. Sciamanna, Opt. Lett. 38 , 703 (2013).

15. M. G. Kovalsky, A. A. Hnilo, and J. R. Tredicce, Opt. Lett. 36, 4449 (2011).

16. N. Marsal, V. Caullet, D. Wolfersbergerm, and M. Sciamanna, Opt. Lett. 39, 3690 (2014).

17. E. Mercier, A. Even, E. Mirisola, D. Wolfersberger, and M. Sciamanna, Phys. Rev. E 91, 042914 (2015).

18. F. Selmi, S. Coulibaly, Z. Loghmari, M. G. Clerc, and S. Barbay, Phys. Rev. Lett. 116, 013901 (2016).

19. A. N. Pisarchik, R. Jaimes-Reategui, R. Sevilla-Escoboza, G. HuertaCuellar, and M. Taki, Phys. Rev. Lett. 107, 274101 (2011).

20. J. Zamora-Munt, B. Garbin, S. Barland, M. Giudici, J. R. R. Leite, C. Masoller, and J. R. Tredicce, Phys. Rev. A 87, 035802 (2013).

21. S. Residori, Phys. Rep. 416, 201 (2005).

22. Further details can be found on the web site of the manufacturer.

23. P. G. de Gennes and J. Prost, The Physics of Liquid Crystals, 2nd ed. (Clarendon, 1993).

24. R. Neubecker, G.-L. Oppo, B. Thuering, and T. Tschudi, Phys. Rev. A 52, 791 (1995).

25. M. G. Clerc, G. Gonzalez-Cortes, and M. Wilson, in Nonlinear Dynamics: Materials, Theory and Experiments, Springer Proceedings in Physics 173, M. G. Clerc and M. Tlidi, eds. (Springer, 2016).

26. M. G. Clerc, G. Gonzalez-Cortes, V. Odent, and M. Wilson, "Optical textures: distinguishing spatiotemporal chaos from amplitude turbulence," Opt. Express, submitted for publication, http://arxiv.org/abs/ 1601.00844 